\title{
IMRT Beam Angle Optimization using Differential Evolution
}

\author{
Joana Dias \\ Inesc-Coimbra and Faculty of Economics \\ University of Coimbra \\ Coimbra, Portugal \\ joana@fe.uc.pt \\ Brígida Ferreira \\ I3N, University of Aveiro \\ Aveiro, Portugal \\ brigida@ua.pt
}

\author{
Humberto Rocha \\ Inesc-Coimbra \\ Coimbra, Portugal \\ hrocha@mat.uc.pt
}

Maria do Carmo Lopes

IPOC-FG, EPE, Coimbra

I3N, University of Aveiro

Aveiro, Portugal

mclopes@ipocoimbra.min-saude.pt

\begin{abstract}
Radiation therapy is one of the main treatments against cancer. Intensity Modulated Radiation Therapy (IMRT) is one type of radiation therapy that allows a high degree of conformity between the radiation intensities and the areas to treat. The planning of a radiation treatment for a given patient is crucial for obtaining the desired goals (being able to destroy cancer cells while preserving the healthy ones). In clinical practice, treatment planning is most of the times based on a lengthy trial-and-error procedure during which the planner interacts with a treatment planning system trying to find a treatment that complies with the medical prescription. One of the first decisions the planner has to make is on the angles to be used to deliver radiation. In clinical practice, most of the times, the number of angles to be used is defined $a$ priori based on the experience of the planner with similar cases. Often, the solution that is used is the equidistant solution, where all angles are equally apart. In this paper we propose the use of Differential Evolution (DE) for determining in an automated way the set of angles that should be used in a given IMRT treatment. Solutions obtained after the DE optimization are then compared with the equidistant solution. Preliminary results considering ten already treated patients at the Portuguese Institute of Oncology of Coimbra IPOCFG are presented.
\end{abstract}

Keywords-Differential Evolution; inverse planning; radiation therapy; optimization

\section{INTRODUCTION}

Cancer is considered by the World Health Organization as one of the main threats for health and human development, being nowadays responsible for about $20 \%$ of deaths in Europe. Radiation therapy, chemotherapy and surgery are the main treatment options, many times used concomitantly. About half of all cancer patients will be submitted to radiation therapy sometime during their illness. Radiation therapy tries to kill cancerous cells by delivering radiation to the areas to treat, while at the same time preserving healthy cells that will also be damaged by radiation. There are many different types of radiation therapy techniques. In this paper we will focus on Intensity Modulated Radiation Therapy (IMRT). In IMRT, high energy photon beam radiation is generated by a linear accelerator mounted on a gantry that can rotate around a central axis parallel to the couch where the patient lays. The rotation of the couch combined with the rotation of the gantry allows irradiation from almost any angle around the tumor. Nevertheless, most of the times, only coplanar treatments are considered.

The head of the gantry has a multileaf collimator (Fig. 1). IMRT is one technique of radiation therapy, where the modulation of the radiation intensity is enabled by the controlled movement of the multiple leaves of the collimator. The leaves are capable of blocking radiation. By changing the position of the leaves from a given angle during the radiation delivery it is possible to achieve intensity modulated radiation profiles. Not only the area to be irradiated is adjusted to the tumor shape, but also the radiation intensity is modulated to better irradiate the tumor, sparing as most as possible adjacent healthy cells. In conceptual terms, we can interpret a radiation beam as being composed by a grid of smaller beamlets of independent intensities.

The planning of a given IMRT treatment is based on the patients' CT images, where the medical doctor delineates all important structures that have to be taken into account during planning (Fig. 2). Important structures to consider are the areas to treat (usually denominated Planning Target Volumes - PTVs), and all the organs to spare (Organs at Risk - OARs). The set of all patients' CT images allow a discretization of the patient into voxels.

For each given patient, the radiation oncologist will also determine the medical prescription that stipulates the radiation doses that should be deposited in the PTVs, and the doses that are acceptable for OARs (see Table 1 for an 
example). For each given patient, there is no guarantee that the medical prescription can be totally fulfilled.

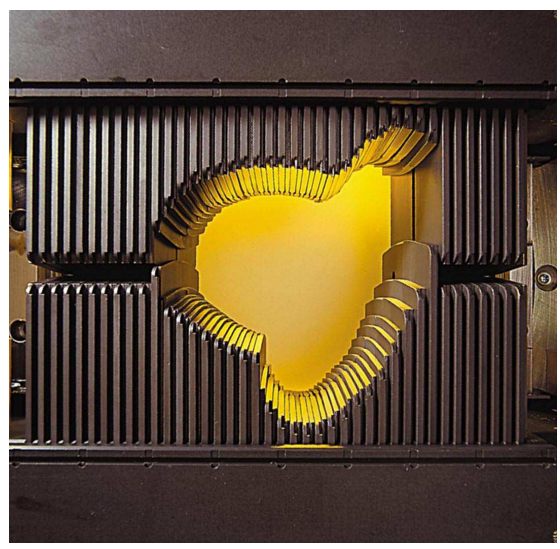

Fig. 1. Multileaf collimator (Image courtesy of Varian Medical Systems, Inc. All rights reserved.)

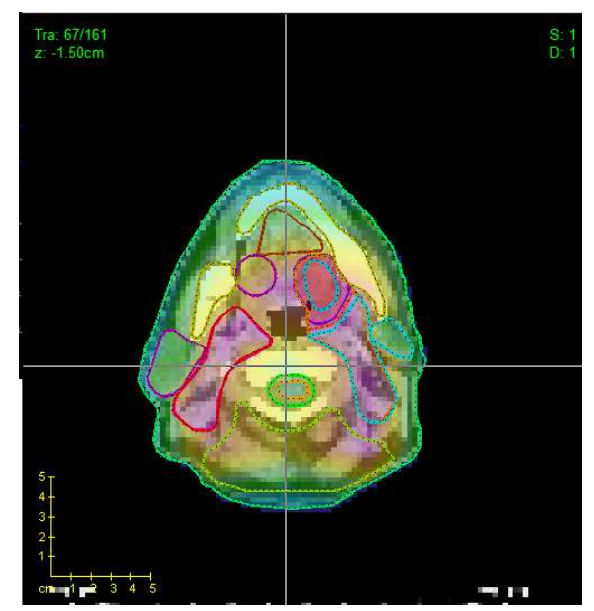

Fig. 2. Example of a patient's CT slice, with several structures delineated.

Depending on organ functionality, OARs can be classified as being serial or parallel organs. Serial organs are such that even if only a small percentage of the organ is over-irradiated the whole organ's functionality is jeopardized (spinal cord, for instance). This is why it is necessary to guarantee that the maximum dose received anywhere within the organ has to be upper bounded. Other organs can still perform their function even if a small percentage of the organ is damaged (lung, for instance). In this case, a dose-volume condition has to be preserved and can be expressed by guaranteeing that the mean dose received is not above a given threshold.

Different IMRT treatment modalities can be considered, like step-and-shoot, dynamic IMRT or Arc Therapy. IMRT treatment planning can be thought as being composed of three sequential and interrelated stages: a. deciding the number of beam angles and their directions (BAO - Beam Angle Optimization); b. calculating the optimal radiation intensity map to be delivered to the patient from every beam direction (FMO - Fluence Map Optimization); c. determining the movement of the multileaf collimator's leaves to deliver the optimal beamlet intensities (Leave Sequencing Problem). We will consider the two first planning stages, and BAO in particular, in step-and-shoot coplanar IMRT.

TABLE I. EXAMPLE OF PRESCRIBED DOSES

\begin{tabular}{cccc}
\hline Structure & Mean dose & Maximum Dose & Prescribed Dose \\
\hline Spinal cord & - & $45 \mathrm{~Gy}$ & - \\
Brainstem & - & $54 \mathrm{~Gy}$ & - \\
Left parotid & $26 \mathrm{~Gy}$ & - & - \\
Right parotid & $26 \mathrm{~Gy}$ & - & - \\
PTV1 & - & - & $70.0 \mathrm{~Gy}$ \\
PTV2 & - & - & $59.4 \mathrm{~Gy}$ \\
Body & - & $80 \mathrm{~Gy}$ & - \\
\hline
\end{tabular}

BAO problem is known to be a challenging problem, highly non-convex and with many local minima. Several approaches have been proposed to tackle this problem [1-8].

In clinical practice, the treatment planning process requires the planner to interact with a treatment planning system, trying different sets of objectives and penalties in a trial and error process until a satisfactory dose distribution is obtained. This is a lengthy procedure, giving no guarantees that the best possible solution is reached. In this paper we will try to contribute to the improvement of the treatment planning process by deciding in an automated way which gantry angles to use. We will do this by looking at the BAO problem as an optimization problem, and by using Differential Evolution (DE) to calculate good quality solutions.

In the next section we will describe the BAO problem. In section III we will briefly describe the DE algorithm. Section IV will show some preliminary computational results. Section V will state some conclusions and possible developments.

\section{BEAM ANGLE OPtIMIZATION PROBLEM}

The first decision a planner has to make is to determine which angles should be used for radiation delivery. It is common practice to fix the number of angles a priori, considering previous experiences with similar cases. Usual number of angles used is 5, 7 or 9, depending on the patient and on the location of PTVs and OARs. The planner will try to plan a treatment with the minimum number of angles possible. Increasing the number of angles is only considered if it is the only way of trying to comply with the medical prescription. Increasing the number of angles will also increase the treatment time. This means that fewer patients can be treated per day. Furthermore, from the patient point of view, faster treatments are better because the probability of deviations relatively to the planning CT due to patient's movements decreases. Actually, the position of the patient during treatment is of the utmost importance: it should be as close as possible to the position during the CT scans, since treatment planning is based on CT images.

After fixing the number of angles to $k$, it is then necessary to determine which angles to use. Each angle will belong to $\left.] 0^{\circ}, 360^{\circ}\right]$. Two different approaches to this problem can be considered: we can discretize the set 
$\left.10^{\circ}, 360^{\circ}\right]$, and the $\mathrm{BAO}$ problem can be interpreted as a combinatorial problem where we seek to find the best combination of $k$ angles out of all possible combinations. We can also choose to interpret each variable as a continuous variable. In this case it is interesting to observe that it is not even necessary to consider bounds to the possible variables' values, since all possible values are admissible $\left(-10^{\circ}\right.$, for instance, is equivalent to $\left.350^{\circ}\right)$. Although most of the approaches in the literature choose the first approach, we will choose the latter. A solution to the BAO problem can thus be interpreted as a vector of $k$ continuous values in $] 0^{\circ}, 360^{\circ}$. For ease in the exposition, we will consider that angles in this vector are in ascending order.

To assess the quality of each solution, it is necessary to calculate the absorbed radiation doses in every voxel of the patient, and consider some measure relating the solution with the medical prescription. This can only be done after solving the FMO problem, that will determine the radiation intensities from every angle considered. There are many ways of solving the FMO problem described in the literature, each one of them with pros and cons. It is out of the scope of this paper to discuss the different possible approaches. In our work, we have chosen to use a quadratic programming problem, where a small amount of underdose or overdose is accepted but larger deviations are decreasingly tolerated. The FMO can be defined as [8]:

$\operatorname{Min}_{w} \sum_{i=1}^{V}\left[\underline{\lambda}_{i}\left(T_{i}-\sum_{j=1}^{N} D_{i j} w_{j}\right)_{+}^{2}+\bar{\lambda}_{i}\left(\sum_{j=1}^{N} D_{i j} w_{j}-T_{i}\right)_{+}^{2}\right]$

s. t. $w_{j} \geq 0, j=1, \ldots, k$

where $T_{i}$ is the desired dose for voxel $i, V$ is the total number of voxels, $\lambda_{i}$ and $\bar{\lambda}_{i}$ are the penalty weights of underdose and overdose of voxel $i$, respectively, $(\bullet)_{+}=\max \{0, \bullet\} . D_{i j}$ corresponds to the dose contribution to voxel $i$ from beamlet $j$ with unit intensity, whereas $w_{j}$ represents the intensity (or fluence) of beamlet $j$. The total dose received by the voxel $i$ is then given by $\sum_{j=1}^{N} D_{i j} w_{j}$, using the superposition principle. It can be calculated using several different algorithms. In this work we used CERR's pencil beam algorithm [9]. The dose calculation can take a considerable amount of time, depending on the patient, delineated structures and number of angles considered. The assessment of a single solution is computationally and time demanding.

If we define $\Theta$ as the set of all possible angles, then $\mathrm{BAO}$ problem can be defined as follows:

$$
\min f\left(\theta_{1}, \ldots, \theta_{k}\right)
$$

Subject to $\theta_{1}, \ldots, \theta_{k} \in \Theta$
In our case, $f(\boldsymbol{\theta}), \boldsymbol{\theta}=\theta_{1}, \cdots, \theta_{k} \in \Theta$, is given by considering all beamlets belonging to $\boldsymbol{\theta}$ and only these. By using this function, we are implicitly assuming that we expect that lower values of $f(\boldsymbol{\theta})$ correspond to better dosimetric results for the patient. Actually, this is not always guaranteed, as will be shown in section IV, but in fact we do need an objective function to guide the search process.

BAO problem is non-linear, non-convex and multimodal, being a challenge for most optimization algorithms. Direct search approaches can be considered to tackle this kind of problems. In this paper we apply DE to this problem.

\section{DIFFERENTIAL EVOLUTION}

$\mathrm{DE}$ is a stochastic direct search method, based on a population of solutions, that is able to handle nonlinear, non-differentiable and multimodal objective functions [10]. The algorithm begins with a population of $n$ randomly generated solutions. The main idea behind DE is to evolve the population by taking the difference vector between two solutions and use this vector to perturb another solution. The algorithm only requires two parameters, $F$ and $C R$, whose role will be explained shortly.

Let $\boldsymbol{\theta}_{i}^{g}$ represent the solution $i$ in iteration (generation) $g$, and $\boldsymbol{\theta}_{i}^{g}[j]$ represent the $j^{\text {th }}$ element of vector $\boldsymbol{\theta}_{i}^{g}$. DE's strategy can be understood as a sequence of mutation, crossover and selection operators, described as follows [10]:

- Mutation: A mutant vector $\boldsymbol{v}$, applied to solution $i$, is calculated by using three different solutions $(a, b$ and $c$ different from $i$ ) as follows:

$$
\boldsymbol{v}=\boldsymbol{\theta}_{a}^{g}+F\left(\boldsymbol{\theta}_{b}^{g}-\boldsymbol{\theta}_{c}^{g}\right), F \in[0,2]
$$

- Crossover: Consider as parents vectors $\boldsymbol{v}$ and $\boldsymbol{\theta}_{i}^{g}$. Then, each angle in solution $\boldsymbol{\theta}_{i}^{g+1}$ will be equal to the angle occupying the same position in $\boldsymbol{\theta}_{i}^{g}$ or $\boldsymbol{v}$, randomly chosen by using a uniform probability distribution, and guaranteeing that at least one angle comes from $\boldsymbol{v}$ by randomly chosen a position $j$ such that $\boldsymbol{\theta}_{i}^{g+1}[j]=v[j]$.

- Selection:Solution $\boldsymbol{\theta}_{i}^{g+1}$ is compared with $\boldsymbol{\theta}_{i}^{g}$. If $f\left(\boldsymbol{\theta}_{i}^{g+1}\right)>f\left(\boldsymbol{\theta}_{i}^{g}\right)$, then $\boldsymbol{\theta}_{i}^{g+1}$ will not replace $\boldsymbol{\theta}_{i}^{g}$ and $\boldsymbol{\theta}_{i}^{g+1} \leftarrow \boldsymbol{\theta}_{i}^{g}$.

The DE algorithm can be briefly described as follows:

1. Generate randomly $n$ solutions. Initialize $i t \leftarrow 1$.

2. $i \leftarrow 1$.

3. Pick randomly three vectors from the population and calculate vector $\boldsymbol{v}$ by using (3). 
4.Calculate $\boldsymbol{\theta}_{i}^{g+1}$ by considering the crossover procedure using $\boldsymbol{\theta}_{i}^{g}$ and $\boldsymbol{v}$ as parents.

5. If $f\left(\boldsymbol{\theta}_{i}^{g+1}\right)>f\left(\boldsymbol{\theta}_{i}^{g}\right)$ then $\boldsymbol{\theta}_{i}^{g+1} \leftarrow \boldsymbol{\theta}_{i}^{g} . i \leftarrow i+1$.

6. If $i \leq n$ then go to 3 . Else $i t \leftarrow i t+1$.

7. If it $>$ maxit then stop. Else go to 2 .

Many variants to this basic algorithm can be thought (see, for instance, [10-14]). In this paper we will apply this basic version of the algorithm to IMRT BAO problem. The only difference between this basic version and the one that was implemented has to do with a specificity of the BAO problem. Regarding clinical outcomes, two angles that differ less than $5^{\circ}$ can be considered as equal. For this reason, after step 4, we guarantee that all adjacent angles are at least $5^{\circ}$ apart.

\section{Computational Results}

The DE algorithm was tested considering ten clinical cases of already treated head-and-neck cancer patients at the Portuguese Institute of Oncology of Coimbra IPOCFG, signalized as complex cases where proper target coverage and organ sparing proved to be difficult to obtain. The medical prescription was similar for all these patients (Table I). For each patient, several PTVs with different dose prescriptions were defined. The simplified OAR list includes the spinal cord, brainstem and parotids. The spinal cord and the brainstem are some of the most critical OARs in the head-and-neck tumor cases, because they are serial type organs. Parotid glands are the largest of the three salivary glands and their irradiation can cause xerostomia (dry mouth due to lack of saliva), decreasing the quality of life of patients due to difficulties to swallow. The parotids are parallel type organs. Treatments with five coplanar beams are considered, since in this case beam direction is critical/fundamental to achieve a good treatment plan.

Our tests were performed on a Intel Core i7 CPU 2.8 GHz 4GB RAM Windows 7 PC. The dose was computed by CERR's pencil beam algorithm [14] (v. 3.2.2, Matlab R2007a). For each of the ten cases, the voxel size was $0.3 \times 0.3 \times 0.3 \mathrm{~cm} 3$. The FMO problem was solved by a trustregion-reflective algorithm (fmincon, Matlab Optimization Toolbox). Each instance of the FMO took from 56 to 350 seconds to be solved. The weights were considered equal to 4 for all PTVs, equal to 2 for all OARs. The prescriptions are depicted in Table I. For different patients, different structures were delineated by the medical doctors, especially considering the PTVs, as can be seen in Table III.

The objective function considered is time demanding. If an automated approach is to be considered in clinical practice, it should be able to produce a good quality solution in a reasonable amount of time, implying that there cannot be too many objective function evaluations. For this reason, and for these preliminary computational experiments, we decided to apply DE considering a very small population of only 4 solutions and a reduced number of iterations (only 50). In this way we limit the number of objective function evaluations to 200. The drawback is that we are loosing some of the exploration capability of the algorithm, and in effect, the solutions that are used in the mutation operator (step 3 of the algorithm) are not randomly chosen: they are all the solutions of the population but the one that is being processed.

The initial set of solutions is constituted by the equidistant solution and three other solutions that are generated by randomly perturbing this equidistant solution (each angle is perturbed by using a normal probability distribution of mean 0 and standard deviation $45^{\circ}$ ).

Taking into account the stochastic behavior of the algorithm, DE was run five times for each patient. Results are then compared with the objective function value of the equidistant solution ( $f_{-}$equi). Table 2 shows the average objective function value over the 5 executions (Average $\left.f_{-} D E\right)$, the objective function standard deviation $(s d)$ and the improvement, in percentage, obtained with the DE approach. The average improvement for all patients is equal to $5.93 \%$, and the standard deviation over the five executions of the algorithm is low. There are, however, huge differences in the objective function value improvement among the patients. This is mainly due to the specificities of each patient, namely the location of the structures of interest (PTVs and OARs), as well as their relative position.

TABLE II. COMPARISON BETWEEN THE EQUIDISTANT SOLUTION AND THE DE CALCULATED SOLUTIONS REGARDING OBJECTIVE FUNCTION VALUES

\begin{tabular}{crrrr}
\hline Patient & \multicolumn{1}{c}{ f_equi } & Average $\boldsymbol{f}_{\mathbf{D}} \boldsymbol{D E}$ & \multicolumn{1}{c}{$\boldsymbol{S \boldsymbol { d }}$} & \% improvement \\
\hline 1 & 207,77 & 202,60 & 2,54 & $2,49 \%$ \\
\hline 2 & 78,80 & 73,85 & 1,08 & $6,28 \%$ \\
\hline 3 & 134,47 & 129,87 & 1,04 & $3,42 \%$ \\
\hline 4 & 169,11 & 160,34 & 1,18 & $5,18 \%$ \\
\hline 5 & 336,42 & 304,69 & 3,07 & $9,43 \%$ \\
\hline 6 & 267,47 & 257,37 & 3,72 & $3,78 \%$ \\
\hline 7 & 41,12 & 37,369 & 0,55 & $9,13 \%$ \\
\hline 8 & 177,90 & 162,77 & 3,84 & $8,50 \%$ \\
\hline 9 & 138,05 & 125,60 & 2,35 & $9,02 \%$ \\
\hline 10 & 195,74 & 191,80 & 2,20 & $2,01 \%$ \\
\hline
\end{tabular}

In this problem, the objective function guides the algorithm towards regions of the search space where we hope better solutions can be found. Nevertheless, in this particular problem, more important than the value of the objective function per se, we are interesting in assessing the clinical impact of these improved solutions. For each of the 10 patients, some dose metrics usually used in clinical practice for plan evaluation were calculated, namely:

- $\quad D_{95}$ - the dose received by $95 \%$ of the PTVs and that should be greater or equal to $95 \%$ of the prescribed dose;

- $D_{\text {mean }}$ - the mean dose received by the whole organ, usually used for parallel OARs (in this case Left and Right Parotids); 
- $D_{\max }$ - the maximum dose received anywhere in the organ, usually used for serial OARs (in this case Spinal Cord and Brainstem).

In an ideal situation we would like to have all PTV1s (primary tumour mass) receiving at least 66.5Gy and all PTV2s (regional lymph nodes) receiving at least 56.05Gy. Considering the OARs, spinal cord should not receive more than $45 \mathrm{~Gy}$ anywhere in the organ. For brainstem, this value increases to 54Gy. Parotids should receive a mean radiation less than $26 \mathrm{~Gy}$.

As for every patient we run the DE algorithm 5 times, generating 5 different solutions, we have chosen to depict the results considering the best and the worst of these solutions, according to the objective function value, comparing them with the equidistant solution. Fig. 3 to Fig. 8 summarize the dosimetric results obtained for the ten patients, considering the equidistant solution and the best and worst DE solutions calculated. Regarding PTVs, different patients have a different number of PTV1 and PTV2 structures delineated. Figures 3 and 4 depict the weighted average dose received (with weights calculated by the relative volumes of each structure).

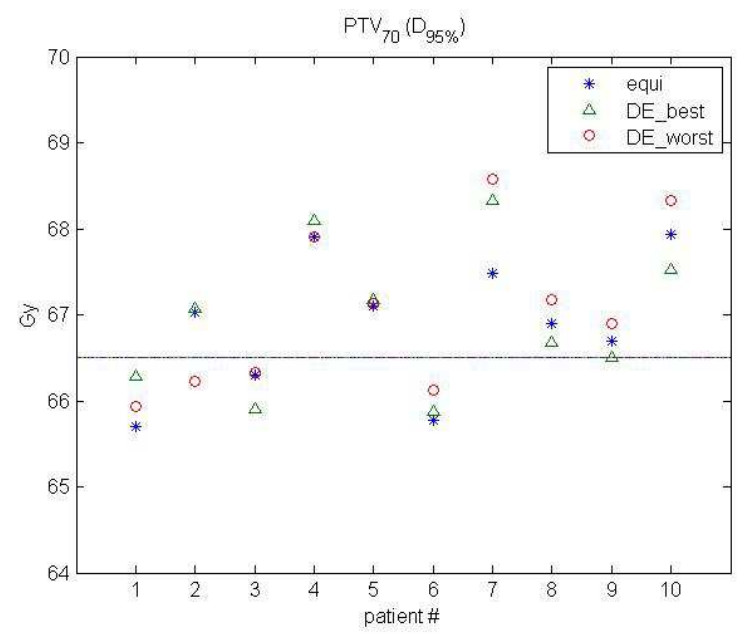

Fig. 3. Dosimetric results considering PTV1.

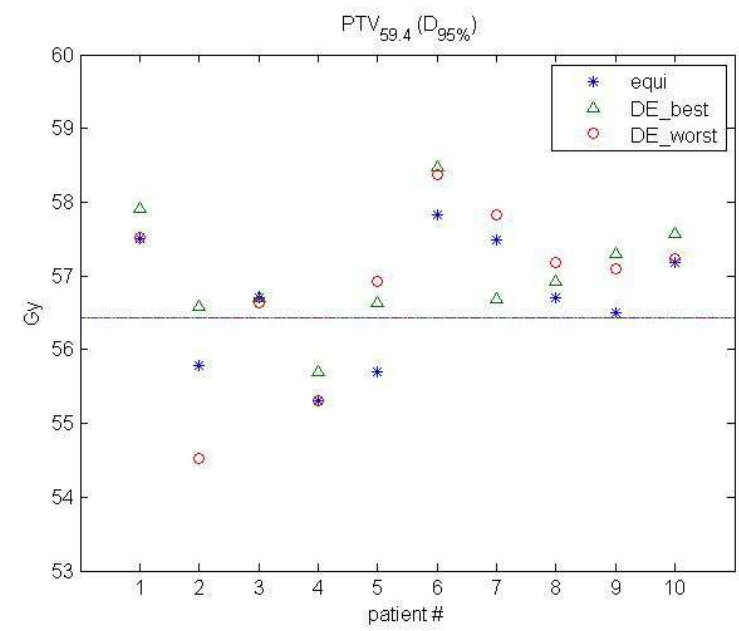

Fig. 4. Dosimetric results considering PTV2.

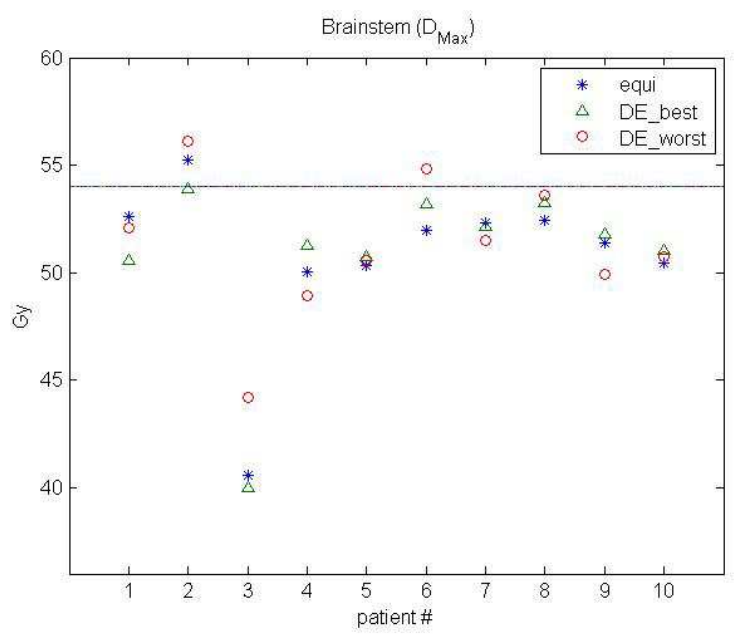

Fig. 5. Dosimetric results considering Brainstem.

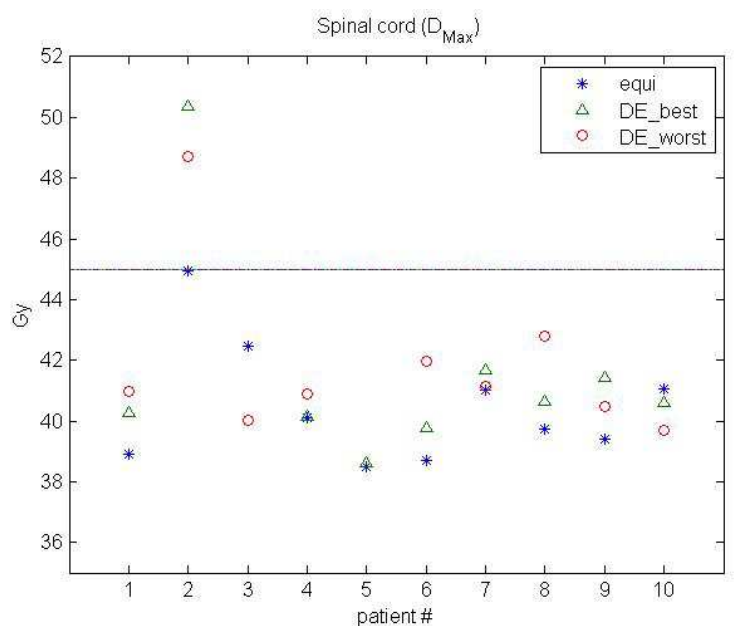

Fig. 6. Dosimetric results considering Spinal Cord.

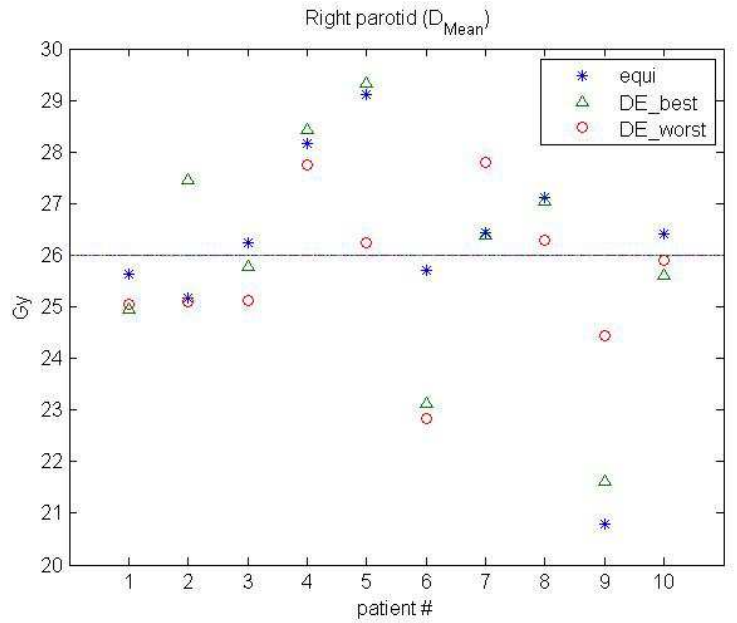

Fig. 7. Dosimetric results considering the Right Parotid. 


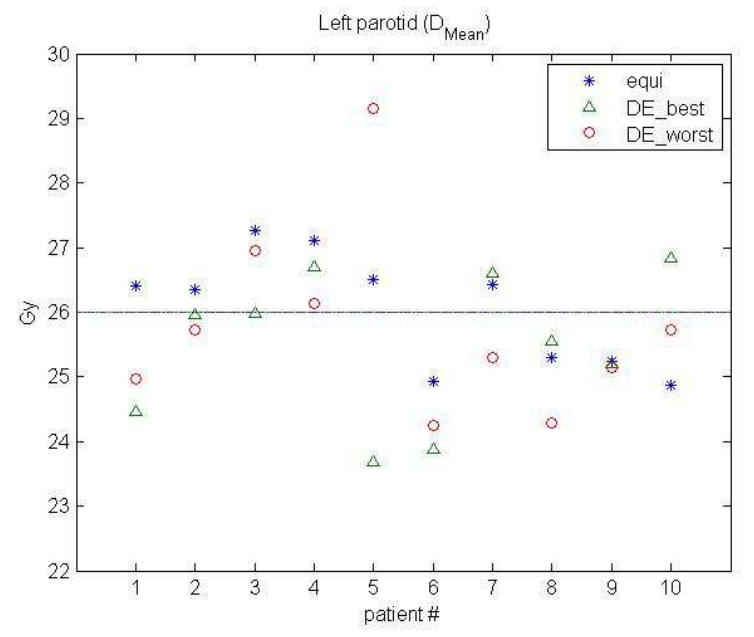

Fig. 8. Dosimetric results considering the Left Parotid.

For PTVs (Fig. 3 and Fig. 4), we would like to have doses above the depicted line. For OARs (Fig. 5 to Fig. 8), we would like to have all values under the depicted lines.

The DE calculated solutions present, for most patients, better planning target coverage and better left and right parotids sparing. This is possibly achieved due to a slightly higher radiation of Spinal Cord, although in all cases but one the treatment complies with the maximum dose allowed.

Detailed dosimetric results are depicted in Table III, where all the planning target structures delineated are discriminated for each patient (PTV1_1, for instance, represents one of the PTV1 structures).

Values in bold correspond to better outcomes of the DE generated solutions.

TABLE III. ASSESSMENT OF THE IMPROVED SOLUTIONS.

\begin{tabular}{|c|l|l|l|l|}
\hline \multicolumn{2}{|c|}{$\begin{array}{l}\text { Patient \& } \\
\text { Structures }\end{array}$} & $\begin{array}{c}\text { Equidistant } \\
\text { solution }\end{array}$ & Best DE solution & \multicolumn{1}{|c|}{$\begin{array}{c}\text { Worst DE } \\
\text { solution }\end{array}$} \\
\hline $\mathbf{1}$ & PTV1_1 & D95=65.70 & D95=66.28 & D95=65.93 \\
& PTV1_2 & D95=65.70 & D95=66.48 & D95=66.28 \\
& PTV1_3 & D95=67.30 & D95=67.78 & D95=67.83 \\
& PTV2_1 & D95=57.50 & D95=57.92 & D95=57.53 \\
& PTV2_2 & D95=57.50 & D95=58.38 & D95=57.93 \\
& Spinal Cord & Dmax=38.90 & Dmax=40.26 & Dmax=40.97 \\
& Left Parotid & Dmean=26.4 & Dmean=24.46 & Dmean=24.97 \\
& Right Parotid & Dmean=25.64 & Dmean=24.94 & Dmean=25.04 \\
& Brainstem & Dmax=52.61 & Dmax=50.58 & Dmax=52.06 \\
\hline \multirow{2}{2}{} & PTV1 & D95=67.03 & D95=67.08 & D95=66.23 \\
& PTV2 & D95=55.78 & D95=56.58 & D95=54.53 \\
& Spinal Cord & Dmax=44.96 & Dmax=50.36 & Dmax=48.71 \\
& Left Parotid & Dmean=26.35 & Dmean=25.96 & Dmean=25.73 \\
& Right Parotid & Dmean=25.18 & Dmean=27.45 & Dmean=25.09 \\
& Brainstem & Dmax=55.25 & Dmax=53.90 & Dmax=56.11 \\
\hline \multirow{3}{*}{} & PTV1 & D95=66.30 & D95=65.9 & D95=66.33 \\
& PTV2 & D95=56.70 & D95=56.70 & D95=56.63 \\
& Spinal Cord & Dmax=42.46 & Dmax=30.65 & Dmax=40.03 \\
& Left Parotid & Dmean=27.26 & Dmean=25.98 & Dmean=26.96 \\
& Right Parotid & Dmean=26.23 & Dmean=25.78 & Dmean=25.13 \\
& Brainstem & Dmax=40.58 & Dmax=40.00 & Dmax=44.23 \\
\hline $\mathbf{4}$ & PTV1_1 & D95=67.90 & D95=68.10 & D95=67.90 \\
& PTV1_2 & D95=67.91 & D95=65.10 & D95=65.10 \\
& PTV2 & D95=55.30 & D95=55.70 & D95=55.30 \\
\hline
\end{tabular}

\begin{tabular}{|c|c|c|c|c|}
\hline & $\begin{array}{l}\text { Spinal Cord } \\
\text { Left Parotid } \\
\text { Right Parotid } \\
\text { Brainstem }\end{array}$ & $\begin{array}{l}\text { Dmax }=40.09 \\
\text { Dmean }=27.11 \\
\text { Dmean }=28.16 \\
\text { Dmax }=50.04\end{array}$ & $\begin{array}{l}\text { Dmax }=40.14 \\
\text { Dmean }=\mathbf{2 6 . 7 1} \\
\text { Dmean }=28.42 \\
\text { Dmax }=51.27\end{array}$ & $\begin{array}{l}\text { Dmax }=40.89 \\
\text { Dmean }=26.14 \\
\text { Dmean }=\mathbf{2 7 . 7 6} \\
\text { Dmax }=\mathbf{4 8 . 9 3}\end{array}$ \\
\hline 5 & $\begin{array}{l}\text { PTV1_1 } \\
\text { PTV1_2 } \\
\text { PTV1_3 } \\
\text { PTV2_1 } \\
\text { PTV2_2 } \\
\text { PTV2_3 } \\
\text { Spinal Cord } \\
\text { Left Parotid } \\
\text { Right Parotid } \\
\text { Brainstem }\end{array}$ & $\begin{array}{l}\text { D95 }=69.30 \\
\text { D95 }=67.10 \\
\text { D95 }=64.90 \\
\text { D95 }=55.10 \\
\text { D95 }=56.30 \\
\text { D95 }=55.70 \\
\text { Dmax }=38.49 \\
\text { Dmean }=26.51 \\
\text { Dmean }=29.10 \\
\text { Dmax }=50.34\end{array}$ & $\begin{array}{l}\text { D95 }=69.30 \\
\text { D95 }=\mathbf{6 7 . 1 8} \\
\text { D95 }=\mathbf{6 5 . 3 0} \\
\text { D95 }=54.30 \\
\text { D95 }=56.10 \\
\text { D95 }=\mathbf{5 6 . 6 3} \\
\text { Dmax }=38.61 \\
\text { Dmean }=\mathbf{2 3 . 6 8} \\
\text { Dmean }=29.34 \\
\text { Dmax }=50.75\end{array}$ & $\begin{array}{l}\text { D95 }=69.30 \\
\text { D95 }=67.13 \\
\text { D95 }=64.70 \\
\text { D95 }=53.90 \\
\text { D95 }=56.30 \\
\text { D95 }=56.93 \\
\text { Dmax }=\mathbf{2 4 . 4 4} \\
\text { Dmean }=29.15 \\
\text { Dmean }=\mathbf{2 6 . 2 3} \\
\text { Dmax }=50.55\end{array}$ \\
\hline 6 & $\begin{array}{l}\text { PTV1_1 } \\
\text { PTV1_2 } \\
\text { PTV1_3 } \\
\text { PTV2_1 } \\
\text { PTV2_2 } \\
\text { Spinal Cord } \\
\text { Left Parotid } \\
\text { Right Parotid } \\
\text { Brainstem }\end{array}$ & $\begin{array}{l}\text { D95 }=68.53 \\
\text { D95 }=65.78 \\
\text { D95 }=65.03 \\
\text { D95 }=57.83 \\
\text { D95 }=55.38 \\
\text { Dmax }=38.72 \\
\text { Dmean }=24.93 \\
\text { Dmean }=25.70 \\
\text { Dmax }=51.96\end{array}$ & $\begin{array}{l}\text { D95 }=68.43 \\
\text { D95 }=\mathbf{6 5 . 8 8} \\
\text { D95 }=63.98 \\
\text { D95 }=\mathbf{5 8 . 4 8} \\
\text { D95 }=\mathbf{5 7 . 4 3} \\
\text { Dmax }=39.78 \\
\text { Dmean }=\mathbf{2 3 . 8 7} \\
\text { Dmean }=\mathbf{2 3 . 1 3} \\
\text { Dmax }=53.21\end{array}$ & $\begin{array}{l}\text { D95 }=\mathbf{6 8 . 6 3} \\
\text { D95 }=\mathbf{6 6 . 1 3} \\
\text { D95 }=64.03 \\
\text { D95 }=\mathbf{5 8 . 3 8} \\
\text { D95 }=\mathbf{5 7 . 4 3} \\
\text { Dmax }=41.95 \\
\text { Dmean }=\mathbf{2 4 . 2 4} \\
\text { Dmean }=\mathbf{2 2 . 8 4} \\
\text { Dmax }=54.81\end{array}$ \\
\hline 7 & $\begin{array}{l}\text { PTV1_1 } \\
\text { PTV1_2 } \\
\text { PTV1_3 } \\
\text { PTV2 } \\
\text { Spinal Cord } \\
\text { Left Parotid } \\
\text { Right Parotid } \\
\text { Brainstem }\end{array}$ & $\begin{array}{l}\text { D95 }=69.18 \\
\text { D95 }=67.48 \\
\text { D95=67.43 } \\
\text { D95=57.48 } \\
\text { Dmax }=41.01 \\
\text { Dmean=26.42 } \\
\text { Dmean }=26.43 \\
\text { Dmax }=52.35\end{array}$ & $\begin{array}{l}\text { D95 }=\mathbf{6 9 . 6 3} \\
\text { D95 }=\mathbf{6 8 . 3 3} \\
\text { D95 }=\mathbf{6 9 . 8 3} \\
\text { D95 }=\mathbf{6 6 . 6 8} \\
\text { Dmax }=41.69 \\
\text { Dmean }=26.60 \\
\text { Dmean }=\mathbf{2 6 . 3 9} \\
\text { Dmax }=\mathbf{5 2 . 1 7}\end{array}$ & $\begin{array}{l}\text { D95 }=69.73 \\
\text { D95 }=68.58 \\
\text { D95 }=69.78 \\
\text { D95 }=67.83 \\
\text { Dmax }=41.14 \\
\text { Dmean }=25.30 \\
\text { Dmean }=27.81 \\
\text { Dmax }=\mathbf{5 1 . 5 2}\end{array}$ \\
\hline 8 & $\begin{array}{l}\text { PTV1_1 } \\
\text { PTV1_2 } \\
\text { PTV1_3 } \\
\text { PTV1_4 } \\
\text { PTV1_5 } \\
\text { PTV1_6 } \\
\text { PTV2_1 } \\
\text { PTV2_2 } \\
\text { Spinal Cord } \\
\text { Left Parotid } \\
\text { Right Parotid } \\
\text { Brainstem }\end{array}$ & $\begin{array}{l}\text { D95 }=69.10 \\
\text { D95 }=68.50 \\
\text { D95 }=66.90 \\
\text { D95 }=65.30 \\
\text { D95 }=66.30 \\
\text { D95 }=67.70 \\
\text { D95 }=56.70 \\
\text { D95 }=56.30 \\
\text { Dmax }=39.72 \\
\text { Dmean }=25.29 \\
\text { Dmean }=27.12 \\
\text { Dmax }=52.45\end{array}$ & $\begin{array}{l}\text { D95 }=\mathbf{6 9 . 4 3} \\
\text { D95 }=\mathbf{6 8 . 5 3} \\
\text { D95 }=66.68 \\
\text { D95 }=\mathbf{6 5 . 7 8} \\
\text { D95 }=\mathbf{6 6 . 8 3} \\
\text { D95 }=\mathbf{6 8 . 0 8} \\
\text { D95 }=\mathbf{5 6 . 9 3} \\
\text { D95 }=56.08 \\
\text { Dmax }=40.66 \\
\text { Dmean }=25.55 \\
\text { Dmean=27.05 } \\
\text { Dmax }=53.27\end{array}$ & $\begin{array}{l}\text { D95 }=\mathbf{6 9 . 4 3} \\
\text { D95 }=\mathbf{6 8 . 6 3} \\
\text { D95 }=\mathbf{6 7 . 1 8} \\
\text { D95 }=\mathbf{6 5 . 7 3} \\
\text { D95 }=\mathbf{6 7 . 3 3} \\
\text { D95 }=67.60 \\
\text { D95 }=\mathbf{5 7 . 1 8} \\
\text { D95 }=\mathbf{5 7 . 0 3} \\
\text { Dmax }=42.78 \\
\text { Dmean }=\mathbf{2 4 . 2 9} \\
\text { Dmean }=\mathbf{2 6 . 3 0} \\
\text { Dmax }=53.62\end{array}$ \\
\hline 9 & $\begin{array}{l}\text { PTV1_1 } \\
\text { PTV1_2 } \\
\text { PTV1_3 } \\
\text { PTV1_4 } \\
\text { PTV2_1 } \\
\text { PTV2_2 } \\
\text { PTV2_3 } \\
\text { Spinal Cord } \\
\text { Left Parotid } \\
\text { Right Parotid } \\
\text { Brainstem }\end{array}$ & $\begin{array}{l}\text { D95 }=69.10 \\
\text { D95 }=66.70 \\
\text { D95 }=66.10 \\
\text { D95 }=66.70 \\
\text { D95 }=57.50 \\
\text { D95 }=57.50 \\
\text { D95 }=56.50 \\
\text { Dmax }=39.39 \\
\text { Dmean }=25.25 \\
\text { Dmean }=20.80 \\
\text { Dmax }=51.40\end{array}$ & $\begin{array}{l}\text { D95 }=69.10 \\
\text { D95 }=66.50 \\
\text { D95 }=65.90 \\
\text { D95 }=65.90 \\
\text { D95 }=57.50 \\
\text { D95 }=57.50 \\
\text { D95 }=\mathbf{5 7 . 3 0} \\
\text { Dmax }=41.44 \\
\text { Dmean }=\mathbf{2 5 . 2 0} \\
\text { Dmean }=21.63 \\
\text { Dmax }=51.81\end{array}$ & $\begin{array}{l}\text { D95 }=69.10 \\
\text { D95 }=\mathbf{6 6 . 9 0} \\
\text { D95 }=65.90 \\
\text { D95 }=65.30 \\
\text { D95 }=57.50 \\
\text { D95 }=57.30 \\
\text { D95 }=\mathbf{5 7 . 1 0} \\
\text { Dmax }=40.49 \\
\text { Dmean }=\mathbf{2 5 . 1 5} \\
\text { Dmean }=24.44 \\
\text { Dmax }=\mathbf{4 9 . 9 3}\end{array}$ \\
\hline 10 & $\begin{array}{l}\text { PTV1_1 } \\
\text { PTV1_2 } \\
\text { PTV1_3 } \\
\text { PTV1_4 } \\
\text { PTV1_5 } \\
\text { PTV2_1 } \\
\text { PTV2_2 } \\
\text { Spinal Cord } \\
\text { Left Parotid } \\
\text { Right Parotid } \\
\text { Brainstem }\end{array}$ & $\begin{array}{l}\text { D95 }=67.93 \\
\text { D95 }=65.63 \\
\text { D95 }=67.08 \\
\text { D95 }=65.28 \\
\text { D95 }=66.38 \\
\text { D95 }=56.53 \\
\text { D95 }=57.18 \\
\text { Dmax }=41.06 \\
\text { Dmean }=24.88 \\
\text { Dmean }=26.41 \\
\text { Dmax }=50.44\end{array}$ & $\begin{array}{l}\text { D95 }=67.53 \\
\text { D95 }=65.63 \\
\text { D95 }=\mathbf{6 7 . 9 8} \\
\text { D95 }=65.28 \\
\text { D95 }=66.28 \\
\text { D95 }=55.88 \\
\text { D95 }=\mathbf{5 7 . 5 8} \\
\text { Dmax }=\mathbf{4 0 . 5 9} \\
\text { Dmean }=26.84 \\
\text { Dmean }=\mathbf{2 5 . 6 2} \\
\text { Dmax }=51.03\end{array}$ & $\begin{array}{l}\text { D95 }=\mathbf{6 8 . 3 3} \\
\text { D95 }=\mathbf{6 6 . 2 8} \\
\text { D95 }=\mathbf{6 7 . 6 3} \\
\text { D95 }=\mathbf{6 5 . 5 3} \\
\text { D95 }=66.23 \\
\text { D95 }=\mathbf{5 6 . 5 8} \\
\text { D95 }=\mathbf{5 7 . 2 3} \\
\text { Dmax }=\mathbf{3 9 . 7 0} \\
\text { Dmean }=25.72 \\
\text { Dmean }=\mathbf{2 5 . 9 0} \\
\text { Dmax }=50.74\end{array}$ \\
\hline
\end{tabular}

As can be seen from Fig. 3 to Fig. 8 and also Table III, the analysis of the dosimetric data of the different solutions is not as straightforward as looking only to the improvement in the objective function value. Actually, for each and every 
patient, it is not possible to find a single solution that is better than the other two for all structures considered.

Looking at patient 1 , we see that de DE solutions obtain a better PTV coverage, guaranteeing the satisfaction of $D_{95}$ values. They also guarantee a better sparing of parotids and brainstem. Considering the spinal cord, the results do not improve, but they are acceptable since $D_{\max }$ is lower than the desired threshold. We can say that, for this patient, results are clearly better for DE solutions, despite the modest improvement of $2.49 \%$ in the objective function value.

Regarding patient 2, we can see that guaranteeing proper sparing of the spinal cord and brainstem and, at the same time, a proper coverage of the PTVs, especially PTV2, is very difficult. This patient should probably be treated with more than 5 angles.

For patient 3, the solutions generated by the DE algorithm seem to be superior to the equidistant solution, considerably improving OARs sparing, without jeopardizing the treatment outcome. For patient 4 , it is also possible to have a better sparing of the parotids, although the coverage of PTV2 is a little bit under what would be desirable.

Patient 5 is one of the patients with the highest improvement in the objective function value. Nevertheless, as can be seen by the results depicted in Table III, the improvements regarding the clinical outcomes are modest. None of the solutions are capable of guaranteeing a proper coverage of PTV2_1. DE solutions seem somewhat better in organs sparing.

Results regarding patient 6 are better for DE solutions, both considering organ sparing and PTV coverage, although not entirely satisfactory for PTV1_3. PTV coverage is much better for DE solutions when looking at patients 7 and 8, and this better coverage does not jeopardize OARs sparing.

Although the objective function improvement for patient 9 is very impressive, this may not necessarily result in a much better expected dosimetric outcome. There are not significant differences between DE solutions and the equidistant solution.

Looking at patient 10 , we see that the DE solutions outperform the equidistant solution, since they guarantee better or equal PTV coverage, but with better organs sparing in general.

In general, we can conclude that solutions generated by the DE procedure usually result in better organ sparing without jeopardizing PTVs coverage. Results have to be carefully analyzed, and it is not sufficient to rely on the improvement of the objective function value since no single objective function has been clinically validated yet and therefore solutions with a better objective function value do not necessary correspond to better solutions from a clinical point of view.

We should remember that we have considered DE with a very small population of solutions, and a very limited number of iterations. These preliminary results show that $\mathrm{DE}$ can be a promising approach to deal with BAO problem.

\section{CONCLUSIONS AND FUTURE WORK}

One of the main advantages of the DE algorithm is the fact that it can be easily parallelized. This will allow the use of populations with more individuals, increasing the exploration capabilities of the algorithm, as well as a greater number of iterations, without increasing too much the computational time. Many of the DE variants that are known from the literature should also be tested. The impact of different objective function values, and the relation between improvements in the objective function and improvements in the clinical outcomes requires further studies. Another way of tackling the expensive objective function evaluation is through the use of surrogate models (see, for instance, [4]). The use of surrogate models allow that only a subset of promising solutions are evaluated by the "true" and computationally expensive objective function, whereas most of the solutions are evaluated by a very fast and hopefully accurate function.

\section{ACKNOWLEDGMENT}

This work was supported by FEDER, COMPETE, iCIS (CENTRO-07-ST24-FEDER-002003) and Portuguese Foundation for Science and Technology under project grants PTDC/EIA-CCO/121450/2010, PEst-OE/EEI/UI308/2014.

\section{REFERENCES}

[1] Li, Y., Yao, J.,Yao, D.: Automatic beam angle selection in IMRT planning using genetic algorithm. Physics in medicine and biology. 49, 1915 (2004)

[2] Li, Y., Yao, D., Yao, J.,Chen, W.: A particle swarm optimization algorithm for beam angle selection in intensity-modulated radiotherapy planning. Physics in medicine and biology. 50, 3491 (2005)

[3] Craft, D.: Local beam angle optimization with linear programming and gradient search. Physics in medicine and biology. 52, N127-N135 (2007)

[4] Dias, J., Rocha, H., Ferreira, B.,Lopes, M.d.C.: A genetic algorithm with neural network fitness function evaluation for IMRT beam angle optimization. Central European Journal of Operations Research. 22, 431-455 (2013)

[5] Dias, J., Rocha, H., Ferreira, B.C.,Lopes, M.d.C. IMRT Beam Angle Optimization Using Dynamically Dimensioned Search. in The International Conference on Health Informatics, IFMBE Proceedings. 2013.

[6] Zhang, H.H., Gao, S., Chen, W., Shi, L., Dâ€苗Souza, W.D.,Meyer, R.R.: A surrogate-based metaheuristic global search method for beam angle selection in radiation treatment planning. Physics in Medicine and Biology. 58, 1933-1946 (2013)

[7] Rocha, H., Dias, J., Ferreira, B.C.,Lopes, M.C.: Selection of intensity modulated radiation therapy treatment beam directions using radial basis functions within a pattern search methods framework. Journal of Global Optimization. 57, 1065-1089 (2013)

[8] Aleman, D.M., Kumar, A., Ahuja, R.K., Romeijn, H.E.,Dempsey, J.F.: Neighborhood search approaches to beam orientation optimization in intensity modulated radiation therapy treatment planning. Journal of Global Optimization. 42, 587-607 (2008)

[9] Deasy, J.O., Blanco, A.I.,Clark, V.H.: CERR: A computational environment for radiotherapy research. Medical Physics. 30, 979-985 (2003)

[10] Storn, R.,Price, K.: Differential Evolution - A Simple and Efficient Heuristic for Global Optimization over Continuous Spaces. Journal of Global Optimization. 11, 341-359 (1997) 
[11] Sun, J., Zhang, Q.,Tsang, E.P.K.: DE/EDA: A new evolutionary algorithm for global optimization. Information Sciences. 169, 249262 (2005)

[12] Qin, A.K.,Suganthan, P.N. Self-adaptive Differential Evolution Algorithm for Numerical Optimization. in The 2005 IEEE Congress on Evolutionary Computation. 2005.

[13] Kovačević, D., Mladenović, N., Bratislav Petrović,Milošević, P.: DEVNS: Self-adaptive Differential Evolution with crossover neighborhood search for continuous global optimization. Computers \& Operations Research.

Price, K.V. Differential evolution: a fast and simple numerical optimizer. in Biennial Conference of the North American. 1996. 